\title{
Optica
}

\section{Fractal propagation method enables realistic optical microscopy simulations in biological tissues: supplementary material}

\author{
Adam K. Glaser ${ }^{1, *}$, Ye Chen $^{1}$, and Jonathan T.C. LiU ${ }^{1}$ \\ ${ }^{1}$ Department of Mechanical Engineering, University of Washington, Seattle, WA, USA \\ *Corresponding author: akglaser@uw.edu
}

Published 1 August 2016

\author{
This document provides supplementary information to "Fractal propagation method enables \\ realistic optical microscopy simulations in biological tissues," http://dx.doi.org/10.1364/ \\ optica.3.000861. (C) 2016 Optical Society of America \\ http://dx.doi.org/10.1364/optica.3.000861.s001
}

\section{THE FRACTAL PROPAGATION METHOD: VALIDITY}

The validity of the fractal propagation method (FPM) relies on the assumption that $\Delta n(x, y, z)<<n_{0}$, (i.e., the turbulent refractive index fluctuations, governed by $D_{f}, l_{c}$, and $\sigma_{n}^{2}$, are much less than the mean background refractive index, $n_{0}$ ) and that $\Delta z$ is sufficiently small, such that diffraction and refraction may be treated independently using the split-step BPM. This results in constraints on the simulation grid resolution, $[\Delta x, \Delta y]$; step size along the optical axis, $\Delta z$; and simulation domain extent $\left[L_{x}, L_{y}, L_{z}\right]$. We start with the spatial resolution of the simulation grid. As discussed in [1], limits on the simulation grid resolution are necessary to ensure the numerical accuracy of the refraction step in the split-step BPM (i.e., application of the 2D phase screens). Numerically, this constraint implies that in order to satisfy the Nyquist criterion, the induced phase difference between any neighboring voxels with a grid resolution must not exceed $\pi$ :

$$
-\pi<\left[\frac{d \phi}{d x}, \frac{d \phi}{d y}\right]<\pi
$$

Here, the induced phase difference, given by $\phi=$ $k_{0} \Delta n(x, y, z) \Delta z$, is assessed in the $x$ and $y$ directions of each $2 \mathrm{D}$ phase screen along the optical axis, $z$. Interestingly, the phase difference is also proportional to the step size $\Delta z$, which must also be chosen properly to ensure that the Nyquist criterion is satisfied when evaluating the Fourier Transforms in the refraction and diffraction steps of the FPM [1].

According to [1], this takes the form of:

$$
\Delta z<\min \left[\frac{2 L_{x} \Delta x}{\lambda}, \frac{2 L_{y} \Delta y}{\lambda}\right]
$$

Due to the fact that both Eqs. S1 and S2 depend on both the step size and grid resolution, the two constraints are inherently linked (i.e., the limits of $\Delta x$ and $\Delta y$ depend on $\Delta z$ and vice versa). In addition, the quantities $d \phi / d x$ and $d \phi / d y$ are dependent on the fractal model parameters and are difficult to calculate before numerically generating an actual in silico fractal refractive index distribution. Therefore, for the FPM, Eqs. S1 and S2 must be checked after an in silico fractal refractive index distribution is generated for a chosen $\Delta x, \Delta y$, and $\Delta z$ to ensure numerical accuracy.

In the context of optical microscopy, it should also be noted that the spatial resolution of the simulation grid is also constrained by the spatial frequencies of the propagating field itself. This can be estimated from the numerical aperture (NA), such that $[\Delta x, \Delta y]<\lambda / 2 \mathrm{NA}$ where $\lambda$ is the wavelength of the propagating beam. However, for tissues represented with a fractal refractive index model, $\mathrm{S} 1$ is typically more restrictive.

Similarly, the simulation grid extent should be chosen to minimize edge effects. When using the BPM, as the field reaches one side of the simulation domain boundary it is transferred to the other side of the domain due to the use of the FFTs, which is physically unrealistic. In addition, the domain size should also be large enough to capture the statistics of the fractal refractive index medium. The first condition is satisfied by properly using a guard band and Tukey window in the frequency and spatial domains respectively. As detailed in [1], the second constraint may be satisfied by ensuring that $L_{x}=L_{y}=L_{z}>10 l_{c}$.

Finally, as discussed in [2] and [3], the validity of the fractal refractive index model for light scattering relies on the Born approximation, in which "weak" scattering dominates (i.e., the scattered field is much less significant than the incident field) [2]. This is similar to the Rytov approximation commonly used in 
the atmospheric-turbulence literature, which describes "weak" and "strong" turbulence based upon a "scintillation index" [4]. This threshold for "weak" scattering was rigorously investigated using FDTD simulation by Capoglu et al. [3]. Here, we use the limiting criteria derived from that study, which states that for a 3D medium:

$$
\sigma_{n}^{2}\left(n_{0} k_{0} l_{c}\right)<<1
$$

where it is also assumed that the correlation length of the medium is less than the mean-free-path of light in the medium, i.e. $l_{c}<1 / \mu_{s}$. It should be noted that these criteria are not concrete limits, but rather estimates of the conditions necessary for numerical accuracy, and that additional expressions for the constraints described in this section exist within the literature [4].

\section{EXPERIMENTAL SETUP}

Fig. S1 shows a detailed schematic of the experimental setup. The light source was a single-mode fiber-coupled (SMF) diode laser (Coherent Laser, Inc., Santa Clara, CA) at $550 \mathrm{~nm}$. For Gaussian illumination, the point source with a $1 / e^{2}$ focusing NA of 0.12 was collimated and focused into tissues without magnification through a pair of matched aspheric lenses with a $25 \mathrm{~mm}$ focal length (NT49-660, Edmund Optics, Barrington, NJ). For Bessel illumination, an axicon with a 20 degree base angle (Thorlabs, AX2520-A, Newton, NJ) was utilized instead of the second aspheric lens in the illumination path. Tissues were placed on a hemispherical solid immersion lens (SIL) with a refractive index of $n=1.45$, which provided index matching to allow the incident beams to focus into the specimens with minimal aberrations. For tissue preparation, small segments $(\sim 2$ mm length) of mouse esophageal mucosa ( 75 $\mu$ m thick) were obtained from a euthanized mouse and rinsed thoroughly with $1 \times$ PBS. We chose to image thin mouse esophagus tissues in order to minimize background due to tissue scattering. This ensured that speckle noise and background (due to scattering) did not overwhelm our ability to discern and quantify the main lobe of the beams. Tissues dissected from mouse esophagi were cut open and the lumenal surface was placed towards the incident beams. As shown in Fig. S1, these fresh tissues were flattened and adhered to the bottom of the coverslip by surface tension, such that we could image the foci of Gaussian and Bessel beams after the beams propagated through the tissue. The gap between the SIL surface and the coverslip was filled with saline buffer.

\section{QUANTIFICATION OF BEAM METRICS}

The quantification metrics utilized in this study are analogous to those of a recent study by Chen et. al. which investigated the steering and distortion of Gaussian and Bessel beams in biological media [5]. These metrics are summarized here.

\section{A. Beam steering}

The motion of the Gaussian and Bessel beam foci for both experiments and FPM simulations is defined as the random "displacement" of either:

1. For experiments: $N$ snap-shot images of a the focused Gaussian or Bessel beam taken at different times, or:

2. For FPM simulations: $N$ independent simulations through different refractive index distributions with the same fractal parameters $\left(D_{f}, l_{c}, n_{0}\right.$, and $\left.\sigma_{n}^{2}\right)$.

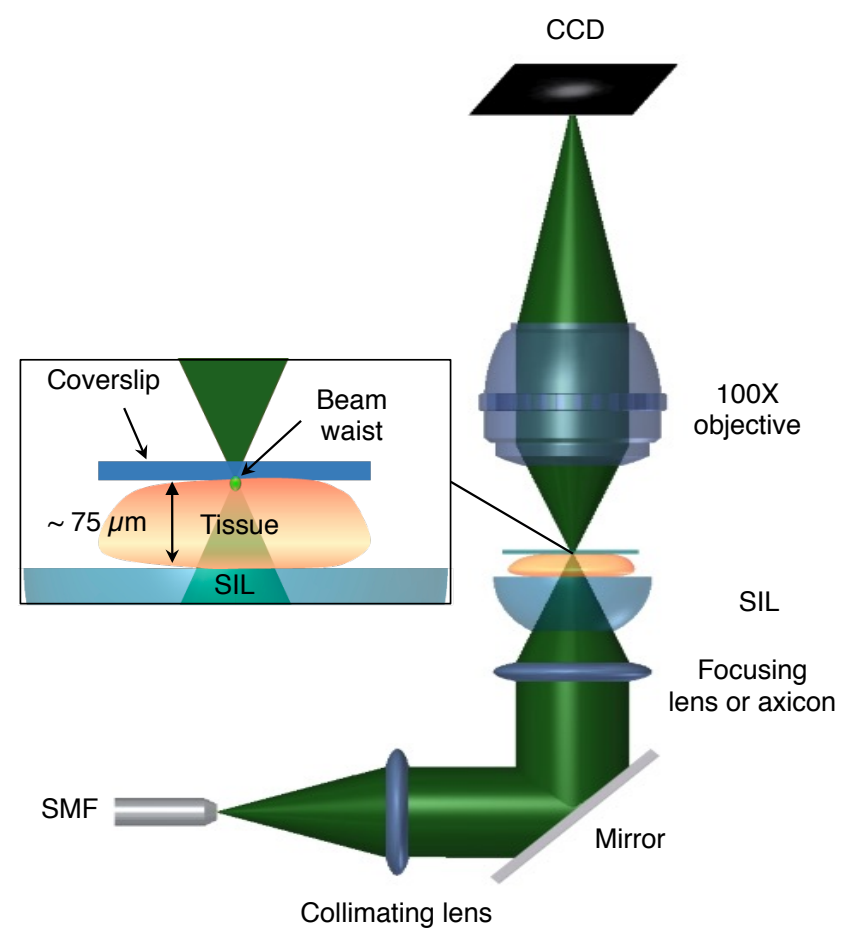

Fig. S1. The experimental setup for the beam steering and distortion measurements is shown.

The "displacement" is quantified as the distance between the intensity-weighted centroid of each experimental image or FPM simulation and that of an unperturbed beam.

\section{B. Beam distortion}

The distortion of the main lobe of the focused Gaussian and Bessel beams is defined as the standard deviation of a "distortion residual" between each experimental snap-shot image or FPM simulation of a beam with respect to an unperturbed beam profile. The process for calculated this distortion residual is summarized below:

1. For each individual snap-shot image or FPM simulation of a focused beam, a 2D Gaussian fit is performed utilizing a least-squares fitting algorithm to obtain four fitting parameters: the centroid location of the focused beam, $\left(x_{0}, y_{0}\right)$, the isotropic beam width, $\omega$, and a scalar value, $A_{0}$, corresponding to the intensity of the experimental image or FPM simulation.

2. For each set of $N$ experimental images or FPM simulations, an average Gaussian surface, $f(x, y)$, is used to approximate an unperturbed beam based on the average beam width, $\bar{\omega}$.

3. Each experimental image or simulation is normalized by dividing the original pixel intensities by $A_{0}$ (determined from Step 1) to form a new normalized image, $I_{i}(x, y)$. Since each image, $I_{i}(x, y)$, has a unique position (center coordinate) due to beam steering, each image is aligned such that the centroid of each experimental image or simulation, $I_{i}(x, y)$, matches the centroid of the unperturbed beam, $f(x, y)$.

4. The difference between $f(x, y)$ and $I_{i}(x, y)$ is calculated, and the standard deviation of this residual for pixels within 
the full-width at $10 \%$ maximum of $f(x, y)$ is calculated to represent the distortion of the Gaussian or Bessel beam. Pixels only within the full-width at $10 \%$ maximum are used to restrict the distortion analysis to the main lobe of the Bessel beam (for comparison to a Gaussian beam) and to omit distortions due to system noise (for experiments) and aberrations in the side lobes (for the Bessel beam).

\section{REFERENCES}

1. D. L. Knepp, "Multiple phase-screen calculation of the temporal behavior of stochastic waves," Proceedings of the Ieee 71, 722-737 (1983).

2. J. D. Rogers, A. J. Radosevich, J. Yi, and V. Backman, "Modeling light scattering in tissue as continuous random media using a versatile refractive index correlation function," IEEE J Sel Top Quantum Electron 20, 7000514 (2013).

3. I. R. Capoglu, J. D. Rogers, A. Taflove, and V. Backman, "Accuracy of the born approximation in calculating the scattering coefficient of biological continuous random media," Opt Lett 34, 2679-81 (2009).

4. L. Andrews and R. Phillips, Laser Beam Propagation through Random Media (SPIE - The International Society for Optical Engineering, Bellingham, WA, 2005), 2nd ed.

5. Y. Chen and J. T. C. Liu, "Characterizing the beam steering and distortion of gaussian and bessel beams focused in tissues with microscopic heterogeneities," Biomed Opt Express 6, 1318-30 (2015). 